\title{
Estudo de Sistemas de Codificação do Teste de Wartegg e suas Relações com o Rorschach (R-PAS)
}

\author{
Fernando Pessotto*, 1 \\ Orcid.org/0000-0002-4448-1577 \\ Ricardo Primi ${ }^{2}$ \\ Orcid.org/0000-0003-4227-6745
}

${ }^{1}$ Centro Universitário Salesiano São Paulo, Americana, SP, Brazil

${ }^{2}$ Universidade São Francisco, Itatiba, SP, Brazil

\section{Resumo}

O Teste de Wartegg é composto por 8 quadros contendo pequenos estímulos gráficos, tendo o sujeito que realizar um desenho a partir deles. Mesmo tendo quase 80 anos de existência, ainda apresenta inconsistências no que diz respeito às evidências de validade e consequente, uso para tomada de decisão. A presente pesquisa teve como objetivo propor um novo modo de codificação e interpretação para a técnica e foi dividida em dois estudos. No primeiro verificou-se cinco diferentes sistemas a fim de verificar potenciais variáveis com maior poder discriminativo. No segundo, seis juízes verificaram quais variáveis do Rorschach poderiam ser utilizadas de forma similar no Teste de Wartegg. Por fim o novo sistema proposto apresenta 13 grupos de critérios para classificação, à saber, orientação, localização, tamanho, conteúdo, qualidade dos objetos, qualidade formal, resposta popular, características particulares, códigos especiais, pressão do traço, tipo do traço, repetição, análise de sequência e título.

Palavras-chave: Avaliação psicológica, técnicas de autoexpressão, técnicas gráficas.

\section{A Study of Coding Systems for the Wartegg Test and their Relations with the Rorschach (R-PAS)}

\begin{abstract}
The Wartegg Test is composed of eight squares with small graphical stimuli in which the person must elaborate drawings from them. Although existing for almost 80 years, it still features inconsistencies in terms of validity evidence and, therefore, its use in decision making. This research aimed to propose a new mode for coding and interpretation for the technique and was divided into two studies. In the first, five different systems were identified with the aim of verifying potential variables with greater discriminative power. In the second, six judges verified which Rorschach variables could be used in a similar way in the Wartegg Test. The new system proposed presents 13 groups of classification criteria,
\end{abstract}

* Endereço para correspondência: Centro Universitário Salesiano São Paulo, Campus Americana, Av. de Cillo, 3500, Parque Universitário, Americana, SP, Brasil 13467-600. E-mail: fpessotto@gmail.com e rprimi@uol. com.br

Apoio: Coordenação de Aperfeiçoamento de Pessoal de Nível Superior (CAPES). 
such as orientation, localization, size, content, quality of the objects, form quality, common response, particular characteristics, special codes, line pressure, line type, repetition, sequence analysis and title.

Keywords: Psychological evaluation, self-expression techniques, graphical techniques.

\section{Un Estudio de Codificación de la Prueba de Wartegg y sus Relaciones con el Rorschach (R-PAS)}

\section{Resumen}

La prueba de Wartegg es composta por ocho cuadros que contienen pequeños estímulos gráficos, en los cuales el sujeto tiene que elaborar dibujos. Aunque con casi 80 años de existencia, presenta inconsistencias al respecto de las evidencias de validad y, consecuente, uso en la toma de decisiones, todavía. La presente investigación objetivó proponer un nuevo modo de codificación y interpretación para la técnica y fue dividida en dos estudios. En el primero verifico-se cinco diferentes sistemas de corrección, a fim de verificar potenciales variables con mayor poder discriminativo. En el segundo, seis jueces verificaron cuales variables del Rorschach podrían ser utilizadas de forma similar en la prueba de Wartegg. Finalmente el nuevo sistema propuesto presenta 13 grupos de criterios para clasificación, a saber: orientación, localización, tamaño, contenido, cualidad de los objetos, cualidad formal, respuesta popular, características particulares, códigos especiales, presión del guión, tipo del guión, repetición, análisis de secuencia y titulo.

Palabras clave: Evaluación psicológica, técnicas de auto-expresión, técnicas gráficas.

A avaliação psicológica é um processo fundamentado cientificamente que busca compreender questões do funcionamento psicológico dos indivíduos com o objetivo de orientar, sugerir e sustentar inferências feitas a partir destas informações (Noronha \& Alchieri, 2004). Neste processo são utilizadas diversas técnicas e testes que visam mensurar e explorar variáveis psicológicas, dentre estas, as de autoexpressão, compostas por estímulos pouco estruturados, não ligados a um construto a priori, a fim de favorecer a expressão individual na formulação da resposta (Fensterseifer \& Werlang, 2008; Meyer \& Kurtz, 2006).

As técnicas de autoexpressão buscam gerar compreensão sobre a dinâmica da personalidade, acessando-as sob a perspectiva vivenciada ou percebida pelo sujeito. Pressupõem, portanto, que a demanda externa, caracterizada nos testes por manchas, desenhos, histórias, entre outros, tem relação com o modo interno em que o indivíduo organiza sua percepção, podendo com isso, ser importante em outros contextos além da personalidade, caracterizando o funcionamento psíquico numa perspectiva diferente das classificações sintomáticas descritas nos manuais de classificação de doenças (Fensterseifer \& Werlang, 2008; Villemor-Amaral \& Pasqualini-Casado, 2006).

Segundo Werlang e Cunha (1993), na interação com o estímulo, são revelados materiais internos do indivíduo, indicando equilíbrio ou não, no que diz respeito aos recursos disponíveis na regulação de conflitos. Por serem estímulos pouco estruturados, quase não oferecem subsídios para que o sujeito utilize informações ou respostas convencionais. Anastasi e Urbina (2000) acrescentam que, por não existir uma resposta correta, ou adequada socialmente, o indivíduo tem pouca inibição ao responder e, consequentemente, a probabilidade de manipulação dos resultados é menor.

Esta compreensão do homem se contrapõe à tradição psicométrica que valoriza dados quantitativos e, contrariamente, enfatiza informações qualitativas, buscando a identificação de tendências espontâneas, motivadas por necessidades implícitas. Contudo, é oportuno salientar que as 
duas fontes de informações são igualmente importantes (Villemor-Amaral \& Pasqualini-Casado, 2006). Subjacente à toda ação encontra-se a individualidade do sujeito que a exerce e implica um modo particular de percebê-la. A este trâmite, Bellak denominou apercepção (Silva, 1989), sendo definida como o processo pelo qual a experiência é percebida, assimilada e interpretada subjetivamente pelo sujeito (Werlang, 2002).

Silva (1989) explica que a apercepção faz parte de um continuum que vai desde a percepção inteiramente objetiva da realidade até a apercepção extrema que pode implicar a quebra do contato com a realidade assumindo inclusive, caráter patológico, ou seja, uma deformação aperceptiva. Várias percepções acontecem simultaneamente, buscando contemplar a maior parte dos estímulos disponíveis possível, num processo interativo implicando um princípio gestáltico de que o todo é mais que a soma das partes.

Dentre as técnicas de autoexpressão, encontra-se o Teste de Wartegg ou Teste de Complemento de Desenhos, originalmente desenvolvido pelo psicólogo alemão Ehrig Wartegg e apresentado para a comunidade científica pela primeira vez em 1937 na Alemanha, com o nome de Wartegg Zeichentest - WZT (Freitas, 1993; Roivainen, 2009). Trata-se de um teste gráfico expressivo, semiestruturado, que visa verificar aspectos da personalidade, a saber, emoção, imaginação, intelecto e atividade (este último encontrado na literatura brasileira como ação ou vontade). É composto por oito quadrados (Quadros) contendo, cada um, um estímulo impresso, sendo solicitado ao sujeito continuar o desenho formando uma figura em cada quadro. Respectivamente, do Quadro 1 ao 8 encontram-se dispostos um ponto, uma pequena linha ondulada, três linhas ascendentes, um pequeno quadrado negro, duas linhas retas em oposição, duas linhas retas desarticuladas, um pequeno semicírculo pontilhado e um grande semicírculo (Crisi, 2007; Freitas, 1993; Kinget, 1952).

Kinget (1952) salienta que a fundamentação teórica do Teste de Wartegg foi baseada na chamada Ganzheit Psychologie (Psicologia da
Totalidade - tradução literal). Esta teoria concebe o sujeito e sua interação com a tarefa desempenhada enquanto estruturas a serem analisadas. No que diz respeito ao sujeito, seu modo de agir é composto por um conjunto de características de natureza dinâmica que tendem a organizar os estímulos que estejam ao alcance da experiência, sendo a emoção o principal elemento de regulação deste sistema. Sendo assim, as experiências são marcadas pela estrutura individual e, consequentemente, é possível inferir suas características. Segundo Biedma e D'Afonso (1973), a hipótese proposta na tarefa do Teste de Wartegg é de que ao executar a tarefa, o indivíduo o faça de acordo com sua maneira individual de perceber, sentir, associar e agir, revelando desta forma, a dinâmica de sua estrutura psíquica.

Ehrig Wartegg, de acordo com Kinget (1952), teria se baseado na técnica criada por Sander, Phantasie Test, em que buscou verificar empiricamente a Ganzheit Psychologie. Os estímulos que compunham o teste eram linhas irregulares que deveriam ser organizadas pelo sujeito de alguma forma. As produções realizadas favorecem a verificação de diferenças acentuadas referentes aos traços estruturais de cada sujeito. Em complemento, Crisi (2007) e Freitas (1993) relatam que o teste é baseado na Teoria da Gestalt considerando que cada indivíduo deverá apresentar, frente aos estímulos, uma maneira pessoal de perceber, sentir, associar e agir, possibilitando acesso a algumas características de sua estrutura psíquica. Segundo esta teoria as propriedades das partes dependem da sua relação com o todo, ou seja, suas qualidades dependem do lugar, papel e função que têm em relação ao todo.

O Teste de Wartegg foi bem aceito e no que diz respeito ao seu uso, Silva (2008) salienta que ela apresenta algumas vantagens como a rápida aplicação, avaliação e interpretação e, também, o fato dos estímulos serem considerados neutros, não estruturados, favorecendo a aceitação pela maioria dos sujeitos. A autora diz ainda que uma produção gráfica pode favorecer comunicações verbais livres, acrescentando maiores possibilidade de análise. 
Mesmo com estas considerações, Roivainen (2009) relata que o Teste de Wartegg é praticamente desconhecido em países anglo-saxônicos, porém, seu uso é frequente na América Latina, Finlândia, Itália e Alemanha. No Brasil foi amplamente utilizado no contexto organizacional ganhando destaque na seleção, avaliação e acompanhamento de profissionais, conforme salienta Berlinck (2000). Ao lado disso Alves, Alchieri e Marques (2001) verificaram que o Teste de Wartegg ficou em $5^{\circ}$ lugar entre os testes de autoexpressão mais ensinados nos cursos de graduação e ainda no estudo de Noronha, Beraldo e Oliveira (2003) o teste foi apontado como o $5^{\circ}$ mais utilizado pelos psicólogos, sendo portanto, um instrumento de grande importância no contexto brasileiro. Mesmo com este cenário, em 2005, o Teste de Wartegg recebeu parecer desfavorável para uso pelo Conselho Federal de Psicologia por não apresentar estudos, sobretudo no que se refere às evidências de validade e precisão, conforme as diretrizes estabelecidas pela entidade na Resolução 002/2003.

\section{Sistemas de Codificação do Teste de Wartegg}

Ehrig Wartegg (1987) apresenta seu primeiro sistema a que se refere como diagnóstico por camadas indicando as diferentes classificações feitas a partir de cada um dos quadros. Neste sistema a classificação é realizada à partir da qualidade formal, conteúdo, sequência e estrutura cognitiva. Com exceção da sequência, em que se analisa a ordem de confecção dos desenhos, as outras apresentam subdivisões a partir de aspectos do desenho.

O autor ressalta a importância de caráter evolutivo na execução, ou seja, da análise ligada à sequência dos desenhos, exemplificando particularidades de diagnóstico psiquiátrico, dificuldades de aprendizagem, processos seletivos e orientação vocacional caracterizando uma tipologia para cada sujeito envolvendo emoção, imaginação, intelecto e energia (ação). Essas considerações são baseadas em grande parte em interpretações puramente clínicas, embasado em análises qualitativas, nem sempre apresentando justificativas sólidas de interpretação, porém, não se pode negar a importância na elaboração do método, assim como as indicações à que se propõe.

O segundo sistema é apresentado por Kinget $(1952,1991)$ que, em complemento aos estudos de Ehrig Wartegg, buscou conceitualizar as tipologias propostas pelo autor introduzindo um procedimento complementar a fim de possibilitar a investigação de conteúdos verbais associados aos desenhos produzidos. Em seus estudos dedicou particular importância à estrutura, ao protocolo de respostas e a seus elementos constituintes. Para a classificação dos desenhos utilizou como grupos de critérios, conteúdo, traço, composição, detalhes, organização, repetição, duplicação, recorrente, variabilidade, fechamento, orientação, cautela, descontração/ desinteresse, movimento, originalidade, popular, claro/vago e consistência/inconsistência.

Além disso, a pesquisadora sistematizou quatro funções básicas avaliadas pelo Teste de Wartegg, à saber, emoção (expansiva e retraída), imaginação (combinatória e criadora), intelecto (prático e especulativo) e atividade (dinâmica e controladora). A classificação do perfil de personalidade é realizada a partir da combinação dos aspectos apresentados anteriormente, sendo ainda ponderadas diferentes intensidades a cada um deles numa escala de cinco pontos. $O$ estudo destas funções permite identificar os estilos de comportamento que determinam a classificação tipológica em Vital-Emocional, RacionalVolitivo e Integrado. Embora tenha atribuído análises quantitativas, a autora ressaltava a importância da análise qualitativa e empregava seus resultados orientados mais à prática do que para requisitos teóricos (Kinget, 1952).

Outro sistema foi proposto por Biedma e D'Afonso (1973) em que os autores realizaram mudanças na forma de correção e até mesmo na quantidade de estímulos, passando de 8 para 16 quadros. Segundo esta proposta, eram analizados 3 aspectos, a saber, comportamento, situações e tomada de posição. Os autores ressaltam ainda a importância dos valores dos estímulos, 
sinalizando a vantagem no uso de estímulos pré estabelecidos por já possuírem características enquanto arquétipos.

Nesta proposta os autores apresentam um sistema de codificação e consequente interpretação baseado em 3 elementos, a saber, fatores de expressão, integração e representação. Os fatores de expressão indicam, fundamentalmente, o modo de ser do sujeito em que a escolha por certas modalidades expressivas indicam seu tipo de funcionamento. Esta categoria é avaliada por características como clareza, dimensão, dinamismo, localização, espessura, originalidade, pressão e simplicidade. Os fatores de integração dizem respeito às representações arquetípicas dos estímulos levando em consideração o desenho confeccionado a partir dele e sua localização no quadro e são compreendidos enquanto arquétipos simples ou compostos. Por fim, os fatores de representação estão ligados ao conteúdo propriamente dito do desenhos realizados em que os autores apresentam uma lista com mais de 80 possibilidades, dentre elas animais, árvores, carta, comida, fogo, olhos, soldados, túnel, entre outras, com indicações de interpretações para cada uma delas (Biedma \& D’Afonso, 1973).

No Brasil, Freitas (1993) propõe o quarto sistema tendo por base o trabalho de Kinget (1952) em que sustenta a ideia que a técnica possibilita analisar a estrutura da personalidade no que diz respeito às funções básicas como emoção, imaginação, dinamismo e controle. Para a interpretação dos resultados a autora apresenta duas formas, a abordagem projetiva e a abordagem expressiva.

$\mathrm{Na}$ abordagem projetiva avalia-se a relação entre a característica gestáltica/arquetípica e o material produzido pelo sujeito, analisando sua adequação ou não. Estas características são evidenciadas a partir dos significados prévios dos estímulos descritos para cada campo. A classificação das mesmas se dá enquanto rabiscos motores, estéticos ou simbólicos, abstrações decorativas simétricas, decorativas assimétricas, ou técnicas e pinturas ou quadros recebendo estes as classificações realismo (natureza animada, fisionomia, esquematismo e natureza inanimada), objetos, fantasias (realidade fantasiada, histórias de fadas, figuras mitológicas, fantasias livres, fantasmas e ainda a repetição do estímulo (Freitas, 1993).

Por sua vez, a abordagem expressiva considera as variáveis no que se refere à propriedades dos desenhos em si. São dividas em aspectos gráficos (bom, médio ou baixo nível de forma) pressão do lápis (muito forte, forte, suave, muito suave, irregular, variação na pressão), linha (reta, curva, contínua, descontínua, trêmula, reforçada, avanços e recuos, e sombreamento), tamanho e expansão gráfica sendo esta classificada como pequena, grande ou compacta (Freitas, 1993)

Mais recentemente, Crisi (2007) apresentou um novo método de codificação e interpretação para o Teste de Wartegg, justificando que o original, além de apresentar índices de evidência de validade desfavoráveis, apresentava um método de pontuação muito complexo e impreciso, e a fundamentação teórica não sustentava toda a complexidade avaliativa proposta por Ehrig Wartegg, fazendo com que seu uso em práticas clínicas, por exemplo, fosse limitado. Crisi propôs um sistema de codificação, pontuação e interpretação baseado no Rorschach na Escola Romana para o que, além de proporcionar um caráter mais objetivo, possibilita a comparação entre os dois testes.

Assim, propôs nove categorias para a codificação dos resultados, a saber, caráter evocativo, qualidade afetiva, qualidade formal, conteúdo, frequência, fenômenos particulares, movimento, resposta impulso e ordem de sucessão ou execução. $\mathrm{O}$ autor ressalta que, com exceção do caráter evocativo, qualidade afetiva e ordem de sucessão, todas as outras foram estabelecidas a partir das variáveis do Rorschach. Além destas variáveis o autor propõem uma análise clínica à partir dos significados arquétipos dos estímulos baseado em interpretações temáticas ligadas às pranchas do Rorschach (Crisi, 1998, 2007). Recentemente Crisi e Dentale (2016) investigou mais profundamente as categorias de caráter evocative, qualidade formal e qualidade afetiva encontrando diferenças significativas destas variáveis entre grupos de pessoas ansiosas, psicóticas e sem diagnóstico psiquiátrico. 
Mesmo este sistema tendo características psicométricas bem definidas, não foram encontrados estudos de evidências de validade para a técnica, sua fundamentação teórica e validade teórica. Contudo demonstra-se uma sistematização importante na busca por um sistema fidedigno de codificação e interpretação para o Teste de Wartegg. Souza, Primi e Miguel (2007) salientam que, embora muitas interpretações realizadas a partir dos resultados do Teste de Wartegg não encontrem sustentação empírica, algumas variáveis parecem estar associadas a traços de personalidade, sendo assim aconselham novos estudos que possibilitem sua verificação e compreensão.

Gronnerod e Gronnerod (2012) também escrevem que interpretações realizadas a partir de resultados do Teste de Wartegg podem chegar a níveis comparáveis com outros métodos de avaliação. Os autores salientam ainda que não há nenhuma razão para rejeita-lo como um método para avaliação da personalidade, porém, é necessária a construção de um sistema sólido, tradição em pesquisas gerando conhecimento acumulado para sua utilização e indicam a necessidade de novas pesquisas baseadas nos estudos já existentes a fim de fortalecer o método empregado.

Este tipo de sistematização foi empregada por Exner para o Rorschach para o Sistema Compreensivo (SC). Esta sistematização conferiu ao Rorschach-SC o rigor psicométrico necessário, com bom grau de concordância entre juízes em sua codificação e interpretação, boa fidedignidade e variância de erro e dados normativos (Costantino, Flanagan, \& Malgady, 1995; Exner, 1999; Weiner, 2000).

Mais recentemente, Meyer, Viglione, Mihura, Erard e Erdberg (2011), propuseram o Rorschach Perfomance Assessment System (R-PAS), um sistema de codificação e interpretação baseado no Sistema Compreensivo visando a melhora na utilização de algumas variáveis em decorrência da grande variação no número de respostas. Além disso, o novo método buscou a simplificação no processo de aplicação da técnica limitando o número de resposta de 2 à 3 por prancha e, novos agrupamentos de vari- áveis tendo por base evidências psicométricas buscando assim maior assertividade nas interpretações feitas a partir dos resultados.

Meyer e cols. (2011) salientam que o novo sistema pode ser considerado uma abordagem internacional e atualizada da técnica como consequência dos avanços no que diz respeito à cientificidade em relação à avaliação da personalidade. Viglione, Meyer, Mihura, Erard, e Erdberg (2012) complementam que esta atualização tem como objetivo ainda, a simplificação no que diz respeito à aprendizagem da técnica por parte dos psicólogos, neste sentido, as principais variáveis mantiveram os mesmos códigos do Sistema Compreensivo enquanto outras foram adequadas para o aperfeiçoamento do sistema.

Neste sentido a presente pesquisa teve como objetivo o estudo de diferentes sistemas de codificação do Teste de Wartegg verificando similaridades entre os mesmos que pudessem compor um novo sistema de codificação. Para alcançar este objetivo a presente pesquisa foi dividida em dois estudos. O primeiro teve como objetivo verificar quais variáveis de codificação são equivalentes entre cinco sistemas de codificação do Teste de Wartegg, à saber, Biedma e D'Afonso (1973), Crisi (2007), Freitas (1993), Kinget (1952) e Wartegg (1987), e quais são mais discriminativos. O segundo, visou verificar quais variáveis do sistema empregado pelo Rorschach R-PAS podem ser utilizadas como critérios para o Teste de Wartegg.

\section{Estudo 1}

\section{Método}

Materiais. Foram utilizados como fonte de consulta os manuais de aplicação, correção e interpretação do Teste de Wartegg, à saber, Biedma e D'Afonso (1973), Crisi (2007), Freitas (1993), Kinget (1952) e Wartegg (1987).

Procedimentos. Em posse dos manuais estudou-se cada um deles com particular ênfase nos sistemas de codificação, ou seja, atribuição de classificações a partir da descrição dos critérios de classificação de atributos dos desenhos. Posteriormente organizou-se em uma planilha eletrônica as classificações e subclassificações 
possíveis para os desenhos em cada um dos sistemas.

\section{Resultados e Discussão}

Com o objetivo de verificar as semelhanças entre os sistemas, as classificações possíveis para os desenhos foram organizadas em uma planilha juntamente com os sistemas que as continham (Tabela 1). Esta análise se deu baseada na aplicação dos critérios para os atributos dos desenhos. Os significados atribuídos para cada indicador nos sistemas muitas vezes eram diferentes ou atribuídos de significados apenas quando agrupados com outros, portanto optou-se em focar nos critérios de classificação, já que os significados destes indicadores serão objetivo de futuros estudos. Sendo assim estes dados resumem as categorias encontradas nos cinco sistemas sinalizando as repetições ou quais são específicas de alguns sistemas.

Tabela 1

Classificações presentes nos Sistemas de Codificação do Teste de Wartegg

\begin{tabular}{|c|c|c|c|c|c|c|c|c|c|c|c|c|c|}
\hline Sistemas & 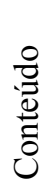 & 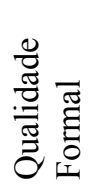 & 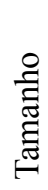 & 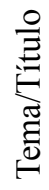 & 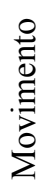 & 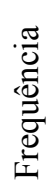 & 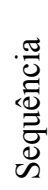 & 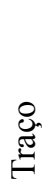 & 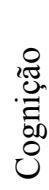 & 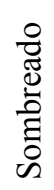 & 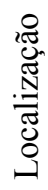 & $\frac{\stackrel{0}{0}}{\sum_{4}^{\frac{1}{4}}}$ & $\frac{\mathscr{d}}{\stackrel{0}{0}}$ \\
\hline Wartegg (1987) & $X$ & $X$ & & & & & $\mathrm{X}$ & & $\mathrm{X}$ & & & & \\
\hline Kinget (1952) & $\mathrm{X}$ & & $\mathrm{X}$ & $\mathrm{X}$ & $\mathrm{X}$ & $\mathrm{X}$ & & $X$ & $\mathrm{X}$ & $\mathrm{X}$ & & & $\mathrm{X}$ \\
\hline Biedma \& D’Afonso (1973) & $\mathrm{X}$ & $\mathrm{X}$ & $\mathrm{X}$ & $\mathrm{X}$ & & $\mathrm{X}$ & & $\mathrm{X}$ & & & $\mathrm{X}$ & & \\
\hline Freitas (1993) & $\mathrm{X}$ & $X$ & $\mathrm{X}$ & $\mathrm{X}$ & $\mathrm{X}$ & & $\mathrm{X}$ & $\mathrm{X}$ & & $\mathrm{X}$ & & $\mathrm{X}$ & \\
\hline Crisi (2007) & $\mathrm{X}$ & $\mathrm{X}$ & $\mathrm{X}$ & $\mathrm{X}$ & $\mathrm{X}$ & $\mathrm{X}$ & $\mathrm{X}$ & & & & & & \\
\hline
\end{tabular}

É possível observar que apenas uma das classificações, o conteúdo, é utilizada nos cinco sistemas estudados. Os critérios para esta pontuação referem-se aos elementos presentes no desenho realizado, indicando em geral, foco de atenção expressos na execução da tarefa. É possível ainda considerar que, por se tratar de um teste gráfico, os conteúdos são considerados elementos projetivos de grande significado para análise do avaliando (Silva, 1989).

A qualidade formal, que diz respeito à adequação do objeto desenhado ao estímulo previamente apresentado no quadro e o tamanho são empregados em quatro dos sistemas pesquisados merecendo também destaque pela sua importância, remetendo, de forma geral, à atributos da qualidade do desenho em relação ao desenho realizado, embora os critérios se diferenciem nos sistemas. Para Biedma e D'Afonso (1973), Crisi (2007) e Freitas (1993) a qualidade formal diz respeito à adequação do desenho ao estímulo inicial enquanto para Wartegg (1987), além da utilização do estímulo, considera-se ainda características como a sobreposição do quadro e o reforço do sinal inicial. Por sua vez, o tamanho refere-se ao espaço do quadro utilizado pelo sujeito.

Também verificado em quarto dos cinco sistemas foi o tema ou título, não tendo sido empregado apenas por Wartegg (1987). Para os demais autores, este critério era utulizado basicamente como dado qualitativo complementando outros indicadores da codificação.

Em seguida, presentes em três sistemas estão movimento, frequência, sequência e traço. $\mathrm{O}$ movimento apresenta-se de forma diferenciada, algumas vezes como subclassificação, outras como código principal. Kinget (1952) apresenta o movimento como código principal dividido em sem movimento, movimento cósmico, movimento mecânico e movimento humano ativo. No sistema de Freitas (1993) o movimento é atribuído no código dinâmica que por sua vez é uma subclassificação do tipo de linha. Por fim, 
Crisi (2007) inclui classificações para movimento dentro do código principal, conteúdo. Esta classificação se dá por movimentos primários e secundários envolvendo subclassificações como sexo, masculino, feminino, indefinido, animal, objeto inanimado e expressões fisionômicas sendo necessário ainda indicar a direção do mesmo nas divisões extensivo, flexivo, bloqueado, duplo, rotatório e indeterminado.

A frequência para os três sistemas é baseada nos estudos observando os desenhos realizados com maior ou menor frequência estando ligado também à classificação originalidade, proposta por Biedma e D'Afonso (1973) e Kinget (1952). Para a análise de sequência, embora os cinco sistemas proponham observar a evolução do sujeito ao longo da prova, apenas em três esta compõem um critério de classificação oferecendo classificações para diferentes tipos de ordem de execução. No que diz respeito ao traço, os sistemas o classificam quanto à pressão/intensidade e ainda o tipo de traço empregado.

Em seguida é possível observar que cognição e sombreamento são empregados em dois sistemas. Cognição refere-se à estrutura cognitiva e, embora citado por outros sistemas, apenas Kinget (1952) e Wartegg (1987) apresentam indicadores ligados à organização cognitiva estando ligados basicamente à complexidade e estrutura envolvida na confecção dos desenhos como detalhes ou ausência deles, desenhos tridimensionais, entre outros. Para sombreamento, tanto Freitas (1993) quanto Kinget (1952) o empregam na presença deste elemento feito no desenho.

Por fim, localização é utilizada por Biedma e D'Afonso (1973) para indicar a área utilizada pelo sujeito na confecção do desenho sendo considerada orientação vertical e horizontal. No que diz respeito ao afeto, Crisi (2007) apresenta uma classificação empregada para a qualidade afetiva apresentada no desenho ou ainda expressa verbalmente.

Embora se tenha estudado os critérios para aplicação dos códigos citados anteriormente, a apresentação detalhada de cada um foge do escopo deste estudo. O que se ressalta é o caráter, muitas vezes, puramente idiográfico para a atribuição dos critérios. Estas informações de cunho clínico são importantes e fazem parte do processo de avaliação, porém Villemor-Amaral $(2006,2009)$ salienta a necessidade da ampliação de evidências de validade para as técnicas de autoexpressão criando-se sistemas que favoreçam maior concordância entre os avaliadores por meio de critério objetivos.

Villemor-Amaral e Pasqualini-Casado (2006) verificaram que existe um número reduzido de estudos destas técnicas, baseados em parâmetros psicométricos. Algumas, por terem grande tradição clínica, são utilizadas nestes tipos de estudos como é o caso do Rorschach no Sistema Compreensivo (Rorschach-SC). Outras, como o Desenho da Figura Humana (DFH), Teste de Apercepção Temática (TAT), Teste de Apercepção Infantil de Figuras (CAT) e o Teste de Wartegg são encontrados com menor frequência na literatura especializada, o que consiste em uma das dificuldades encontradas no que se refere a sistemas confiáveis de avaliação e interpretação.

Lilienfeld, Wood e Garb (2000) já sinalizavam que, para algumas técnicas de autoexpressão, os critérios utilizados para correção e consequente interpretação, muitas vezes se baseiam apenas num julgamento clínico, subestimando o rigor técnico e metodológico de procedimentos psicométricos, o que inviabiliza as generalizações das interpretações pretendidas. Villemor-Amaral (2008) acrescenta que a interpretação feita a partir de técnicas de autoexpressão deve utilizar parâmetros estruturais claros provenientes das teorias. Em concordância, não foram encontrados muitos estudos com o Teste de Wartegg e ainda, alguns apresentavam evidências desfavoráveis para seu uso (Roivainen, 2009; Silva, 2008; Souza et al., 2007).

Neste sentido foram propostas classificações que favorecessem a aplicação de forma clara, ou seja, àquelas que os critérios de classificação eram suficientemente claros na codificação. Como se trata de um estudo exploratório para a criação de um novo sistema, considerou-se importante manter todos os critérios possíveis de serem empregados na codificação dos desenhos. Eventualmente, estudos futuros indicarão àqueles com melhor precisão ou ainda os que não tem 
força de discriminação de traços latentes dos indivíduos.

O sistema final foi composto por 13 critérios, alguns contendo subclassificações. As classificações primárias propostas foram orientação, localização, tamanho, conteúdos, qualidade dos objetivos, qualidade formal, popular, características particulares, códigos especiais, traço, repetição, análise de sequência e título. Com exceção da análise de sequência, cada classificação deve ser atribuída para os 8 quadros, podendo algumas apresentar mais de um código.

\section{Estudo 2}

\section{Método}

Participantes. Participaram deste estudo 6 juízes com experiência em técnicas de autoexpressão sendo 4 doutores e 2 doutorandos. A idade dos juízes variou entre 32 e $41(M=36,83$; $D P=3,31)$ sendo quatro juízes do sexo masculino. Em relação à localidade, quatro atuam no estado de São Paulo, um no Pará e outro em Minas Gerais.

\section{Materiais}

Tabela das variáveis do Teste de Wartegg e Método de Rorschach. Foram desenvolvidas duas planilhas eletrônicas para este estudo. A Planilha 1 continha os 10 grupos de indicadores do Rorschach (R-PAS), à saber, orientação, localização, espaços, conteúdos, qualidade dos objetos, qualidade formal, popular, determinantes, códigos cognitivos e códigos temáticos. Ao clicar sobre cada um deles, redireciona-se para outra aba contendo o indicador e seus critérios no Rorschach (R-PAS). Os juízes tiveram que indicar como cada um dos critérios poderia ser utilizado no Teste de Wartegg. Por exemplo, tomando como base a definição de qualidade formal (FQ) do R-PAS, o juízes deveria dizer de que forma este critério poderia ser utilizado levando em conta a tarefa do Teste de Wartegg.

A Planilha 2 continha os mesmos indicadores, porém, eram apresentados os do Rorschach (R-PAS) e o juiz tinha que indicar qual seria o correspondente no Teste de Wartegg (nesta planilha já inidcada a relação pelos autores deste estudo), tendo ele também, a possibilidade de justificar. Ou seja, os juízes tiveram que verificar a pertinência teórica entre as variáveis indicadas, ou seja, se para o Teste de Wartegg, aquele pode ser um indicador utilizado da mesma forma que no R-PAS oferecendo ainda uma justificativa teórica para sua resposta. Os juízes puderam ainda sinalizar que algum indicador do R-PAS poderia ser utilizado em outro critério de codificação no Wartegg.Para os dois casos, junto às planilhas, encaminhou-se uma breve explicação da tarefa a ser desempenhada no Teste de Wartegg, uma folha de estímulos em branco e um exemplo preenchido afim de sanar dúvidas a respeito e um questionário contemplando questões socioeconômicas e de formação como titulação máxima e prática com as técnicas de autoexpressão.

Procedimentos. Com o trabalho aprovado pelo Comitê de Ética em Pesquisa da Universidade São Francisco, os juízes foram convidados a participar do estudo e após assinatura, por meio eletrônico, do Termo de Consentimento Livre e Esclarecido (TCLE) receberam via email o material da pesquisa. Foram convidados $17 \mathrm{ju}$ ízes, sendo que, oito responderiam à Planilha $1 \mathrm{e}$ nove à Planilha 2. Ao final, aceitaram participar do estudo seis juízes, sendo que quatro responderam à Planilha 1 enquanto dois responderam à Planilha 2.

Plano de análise de dados. As respostas dos juízes foram analisadas qualitativamente considerando as semelhanças indicadas por eles, bem como as considerações elencadas. Neste sentido, foram verificadas as concordâncias entre eles no que diz respeito à quais critérios poderiam ser utilizados no Teste de Wartegg e ianda realizada uma análise do conteúdo das justificativas que foram apresentadas.

\section{Resultados e Discussão}

Para verificar as variáveis potencialmente em comum entre o Teste de Wartegg e o Rorschach (R-PAS) os juízes indicaram, baseados em suas experiências com técnicas de autoexpressão, mais especificamente, com o Rorschach, àquelas que poderiam se adequar à proposta. Na Tabela 2 podem ser verificadas as relações indicadas. 
Tabela 2

Variáveis com Similaridade entre o Teste de Wartegg e o Rorschach (R-PAS) Indicada pelos Juízes

\begin{tabular}{|c|c|c|c|c|c|c|c|c|}
\hline Classificação & código & Juiz 1 & Juiz 2 & Juiz 3 & Juiz 4 & Juiz 5 & Juiz 6 & TOTAL \\
\hline \multirow[t]{4}{*}{ Orientação } & $<$ & 1 & 0 & 1 & 1 & 1 & 1 & 5 \\
\hline & $>$ & 1 & 0 & 1 & 1 & 1 & 1 & 5 \\
\hline & $\mathrm{v}$ & 1 & 0 & 1 & 1 & 1 & 1 & 5 \\
\hline & a & 1 & 0 & 1 & 1 & 0 & 1 & 4 \\
\hline \multirow[t]{3}{*}{ Localização } & $\mathrm{W}$ & 1 & 1 & 1 & 1 & 1 & 1 & 6 \\
\hline & $\mathrm{D}$ & 1 & 1 & 1 & 1 & 1 & 1 & 6 \\
\hline & $\mathrm{Dd}$ & 0 & 0 & 1 & 1 & 1 & 1 & 4 \\
\hline \multirow[t]{2}{*}{ Espaços } & SR & 0 & 0 & 1 & 1 & 0 & 1 & 3 \\
\hline & SI & 1 & 0 & 1 & 0 & 0 & 1 & 3 \\
\hline \multirow[t]{17}{*}{ Conteúdo } & $\mathrm{H}$ & 1 & 1 & 1 & 1 & 1 & 1 & 6 \\
\hline & $(\mathrm{H})$ & 1 & 1 & 1 & 1 & 1 & 1 & 6 \\
\hline & $\mathrm{Hd}$ & 1 & 1 & 1 & 1 & 1 & 1 & 6 \\
\hline & (Hd) & 1 & 0 & 1 & 1 & 1 & 1 & 5 \\
\hline & $\mathrm{A}$ & 1 & 0 & 1 & 1 & 1 & 1 & 5 \\
\hline & (A) & 1 & 0 & 1 & 1 & 1 & 1 & 5 \\
\hline & $\mathrm{Ad}$ & 1 & 0 & 1 & 1 & 1 & 1 & 5 \\
\hline & (Ad) & 1 & 0 & 1 & 1 & 1 & 1 & 5 \\
\hline & An & 1 & 1 & 1 & 1 & 1 & 1 & 6 \\
\hline & Art & 1 & 1 & 1 & 1 & 1 & 1 & 6 \\
\hline & Ay & 1 & 1 & 1 & 1 & 1 & 1 & 6 \\
\hline & $\mathrm{Bl}$ & 1 & 0 & 1 & 1 & 1 & 1 & 5 \\
\hline & $\mathrm{Cg}$ & 1 & 1 & 1 & 1 & 1 & 1 & 6 \\
\hline & Ex & 1 & 1 & 1 & 1 & 1 & 1 & 6 \\
\hline & $\mathrm{Fi}$ & 1 & 1 & 1 & 1 & 1 & 1 & 6 \\
\hline & $\mathrm{Sx}$ & 1 & 1 & 1 & 1 & 1 & 1 & 6 \\
\hline & $\mathrm{NC}$ & 1 & 1 & 1 & 1 & 1 & 1 & 6 \\
\hline \multirow[t]{3}{*}{ Qualidade dos Objetos } & Sy & 1 & 1 & 1 & 1 & 1 & 1 & 6 \\
\hline & $\mathrm{Vg}$ & 1 & 1 & 1 & 1 & 1 & 1 & 6 \\
\hline & $\operatorname{Par}(2)$ & 0 & 0 & 0 & 0 & 0 & 1 & 1 \\
\hline \multirow[t]{4}{*}{ Qualidade Formal } & o & 1 & 1 & 1 & 1 & 1 & 1 & 6 \\
\hline & $\mathrm{u}$ & 1 & 1 & 1 & 1 & 1 & 1 & 6 \\
\hline & - & 1 & 1 & 1 & 1 & 1 & 1 & 6 \\
\hline & $\mathrm{n}$ & 1 & 0 & 0 & 1 & 1 & 1 & 4 \\
\hline
\end{tabular}




\begin{tabular}{|c|c|c|c|c|c|c|c|c|}
\hline Popular & $\mathrm{P}$ & 1 & 1 & 1 & 1 & 1 & 1 & 6 \\
\hline \multirow[t]{15}{*}{ Determinantes } & M & 1 & 1 & 1 & 1 & 1 & 1 & 6 \\
\hline & FM & 1 & 1 & 1 & 1 & 1 & 1 & 6 \\
\hline & $\mathrm{m}$ & 1 & 1 & 1 & 1 & 1 & 1 & 6 \\
\hline & $\mathrm{a}$ & 1 & 1 & 1 & 0 & 1 & 1 & 5 \\
\hline & $\mathrm{p}$ & 1 & 1 & 1 & 0 & 1 & 1 & 5 \\
\hline & $\mathrm{FC}$ & 0 & 0 & 0 & 0 & 0 & 0 & 0 \\
\hline & $\mathrm{CF}$ & 0 & 0 & 0 & 0 & 0 & 0 & 0 \\
\hline & $\mathrm{C}$ & 0 & 0 & 0 & 0 & 0 & 0 & 0 \\
\hline & $\mathrm{C}^{\prime}$ & 1 & 0 & 0 & 0 & 1 & 0 & 2 \\
\hline & $\mathrm{T}$ & 0 & 0 & 1 & 0 & 1 & 1 & 3 \\
\hline & $\mathrm{V}$ & 0 & 1 & 1 & 1 & 1 & 0 & 4 \\
\hline & $\mathrm{Y}$ & 0 & 1 & 1 & 1 & 1 & 0 & 4 \\
\hline & FD & 1 & 1 & 1 & 0 & 1 & 1 & 5 \\
\hline & $\mathrm{r}$ & 0 & 0 & 0 & 0 & 1 & 0 & 1 \\
\hline & $\mathrm{F}$ & 1 & 0 & 1 & 1 & 1 & 0 & 4 \\
\hline \multirow[t]{8}{*}{ Códigos Cognitivos } & Level I & 0 & 1 & 1 & 0 & 0 & 1 & 3 \\
\hline & Level II & 0 & 1 & 0 & 0 & 0 & 1 & 2 \\
\hline & DV (I e II) & 1 & 0 & 1 & 0 & 1 & 1 & 4 \\
\hline & DR (I e II) & 1 & 0 & 1 & 0 & 1 & 1 & 4 \\
\hline & PEC & 1 & 0 & 1 & 0 & 1 & 1 & 4 \\
\hline & INC (I e II) & 1 & 1 & 1 & 0 & 1 & 1 & 5 \\
\hline & FAB (I e II) & 1 & 1 & 1 & 1 & 1 & 1 & 6 \\
\hline & CON & 1 & 1 & 1 & 1 & 1 & 1 & 6 \\
\hline \multirow[t]{11}{*}{ Códigos temáticos } & ABS & 1 & 1 & 1 & 0 & 1 & 1 & 5 \\
\hline & PER & 1 & 1 & 1 & 0 & 1 & 1 & 5 \\
\hline & $\mathrm{COP}$ & 1 & 1 & 1 & 0 & 1 & 1 & 5 \\
\hline & MOR & 1 & 1 & 1 & 0 & 1 & 1 & 5 \\
\hline & MAH & 1 & 0 & 1 & 0 & 1 & 1 & 4 \\
\hline & MAP & 1 & 1 & 1 & 0 & 1 & 1 & 5 \\
\hline & GHR & 1 & 1 & 1 & 1 & 1 & 1 & 6 \\
\hline & PHR & 1 & 1 & 1 & 1 & 1 & 1 & 6 \\
\hline & AGM & 1 & 1 & 1 & 0 & 1 & 1 & 5 \\
\hline & $\mathrm{AGC}$ & 1 & 1 & 1 & 1 & 1 & 1 & 6 \\
\hline & ODL & 1 & 1 & 1 & 0 & 1 & 1 & 5 \\
\hline
\end{tabular}


Dos 68 códigos, divididos em 10 agrupamentos analisados, 27 foram considerados comuns entre os instrumentos por todos os juízes, 20 por 5 juízes e 10 por 4 dos juízes. Estas variáveis foram consideras relevantes para o novo sistema em desenvolvimento para o Teste de Wartegg considerando sua pertinência no sistema do Rorschach (R-PAS). Os demais, ou seja, 11 códigos que foram considerados pertinentes por 3 ou menos juízes, não foram considerados para o novo sistema. Além das indicações apresentadas na Tabela 2, foram considerados ainda os comentários feitos pelos juízes em diversas variáveis.

Verificar a similaridade entre os sistemas pode favorecer a criação de um novo sistema para o Teste de Wartegg baseado em um instrumento já bastante utilizado e pesquisado como o Rorschach conforme sinalizam Gronnerod e Gronnerod (2012). Além disso, pode facilitar estudos entre os instrumentos como evidências de validade e validade consequencial conferindo ao Teste de Wartegg melhor grau de concordância entre os juízes, na interpretação e na fidedignidade conforme verificaram Costantino et al. (1995), Exner (1999) e Weiner (2000) para o Rorschach.

Após esta análise e as considerações do Estudo 1, evidenciaram-se do Teste de Wartegg 13 critérios de classificação, ou seja, nos sistemas estudados foram encontrados 14 critérios a serem utilizados na codificação da ferramenta. Os critérios propostos foram orientação, localização, tamanho, conteúdo, qualidade dos objetos, qualidade formal, resposta popular, características particulares, códigos especiais, traço (pressão do traço e tipo do traço), repetição, análise de sequência e título.

\section{Considerações Finais}

O Estudo 1 desta pesquisa teve como objetivo estudar cinco sistemas de codificação do Teste de Wartegg, à saber, Biedma e D'Afonso (1973), Crisi (2007), Freitas (1993), Kinget (1952), e Wartegg (1987), a fim de verificar seus critérios de classificação para os desenhos reali- zados tendo sido verificados 13 conjuntos de variáveis utilizados. Já o Estudo 2, buscou verificar quais variáveis do Rorschach (R-PAS) poderiam ser utilizadas para o Teste de Wartegg tendo por base suas tarefas. Pode-se verificar algumas variáveis em comum, bem como outras utilizadas apenas por um dos sistemas, conforme descrito nos resultados.

Mesmo com vários sistemas, o instrumento ainda apresenta poucos estudos psicométricos o que dificulta a verificação com maiores detalhes de similaridades nos diferentes sistemas, porém, grande parte dos critérios estudados, apresentam quase que exclusivamente conteúdo idiográfico o que dificulta a codificação, e posterior interpretação, similar por diferentes juízes. O sistema que hora se propõe configura-se como um início de uma reestruturação do instrumento devendo ser estudado em pesquisas futuras contemplando evidências de validade e fidedignidade, buscando melhor compreender as habilidades latentes intrínsecas aos indicadores da técnica.

Neste sentido a presente pesquisa possibilitou identificar um conjunto de critérios a ser utilizado na codificação dos desenhos realizados no Teste de Wartegg. A composição final apresenta 13 conjuntos de critérios com subcritérios, sendo eles, orientação, localização (vertical, horizontal, central e total), tamanho (pequeno, grande ou médio), conteúdo (figura humana inteira, figura para-humana inteira, parte de fiugra humana, parte de figura para-humana, animal inteiro, pára-animal inteiro, parte de animal, parte de pára-animal, anatomia, arte, antropologia, sangue, vestuário, explosão, fogo, sexo, outros), qualidade dos objetivos (síntese ou vago), qualidade formal (ordinário, incomum, distorcido), popular, características particulares (movimento humano, movimento animal, movimento inanimado, profundiade [sombreado], textura, profundidade [não relative ao sombreado], códigos especiais (combinação fabulada, contaminação, representação abstrata, resposta personalizada, movimento cooperative, mórbido, mutualidade de autonomia saudável, mutualidade de autonomia patológico, boa representação humana, má representação humana, movimento agressivo, 
conteúdo agressivo e linguagem dependente), tipo de traço - pressão (fraco, moderado, forte) tipo de traço - linha (continua, descontínua, trêmula ou reforçada), repetição, análise de sequência e título.

\section{Referências}

Alves, I. C. B., Alchieri, J. C., \& Marques, K. (2001). Panorama geral do ensino das técnicas de exame psicológico no Brasil (pp. 10-11). Trabalho apresentado no I Congresso de Psicologia Clínica - Programas e Resumos. São Paulo, SP: Universidade Presbiteriana Mackenzie.

Anastasi, A., \& Urbina, S. (2000). Testagem psicológica. Porto Alegre, RS: ArtMed.

Berlinck, V. (2000). O teste de completamento de desenos Wartegg em universitarios de São Paulo (Dissertação de mestrado). Universidade de São Paulo, SP, Brasil.

Biedma, C. J., \& D'Afonso, P. G. (1973). A linguagem do desenho - Teste de Wartegg - Bidema. São Paulo, SP: Mestre Jou.

Costantino, G., Flanagan, R., \& Malgady, R. (1995). The history of the Rorschach: Overcoming bias in multicultural projective assessment. Rorschachiana, 20(1), 148-171. doi: http://doi. org/10.1027/1192-5604.20.1.148

Crisi, A. (1998). Introducción al Test de Completamiento de Dibujo Wartegg (W.D.C.T.). Buenos Aires: Asociación Argentina de Estudio e Investigación en Psicodiagnóstico.

Crisi, A. (2007). Manuale del test di Wartegg. Norme per la raccolta, la siglatura e l'interpretazione (2. ed.). Roma: Magi Edizione.

Crisi, A., \& Dentale, F. (2016). The Wartegg Drawing Completion Test: Inter-rater Agreement and Criterion Validity of Three New Scoring Categories. International Journal of Psychology and Psychological Therapy, 16(1), 83-90.

Exner, J. E. (1999). Manual de interpretação do Rorschach para o sistema compreensivo. São Paulo, SP: Casa do Psicólogo.

Fensterseifer, L., \& Werlang, B. S. G. (2008). Apontamentos sobre o status científico das técnicas projetivas. In A. E. de Villemor-Amaral \& B. S. G. Werlang (Eds.), Atualizações em Métodos Projetivos para Avaliação Psicológica (pp. 15-33). São Paulo, SP: Casa do Psicólogo.
Freitas, A. M. L. (1993). Guia de Aplicação e Avaliação do Teste Wartegg. São Paulo, SP: Casa do Psicólogo.

Gronnerod, J. S., \& Gronnerod, C. (2012). The Wartegg Zeichen Test: A literature overview and a meta-analysis of reliability and validity. Psychological Assessment, 24(2), 476-489. doi: http:// doi.org/10.1037/a0026100

Kinget, G. M. (1952). The drawing-completion test: A projective technique for the investigation of personality, based on the Wartegg test blank. New York: Grune \& Stratton.

Kinget, G. M. (1991). O teste de Completamento de Figuras. In E. F. Hammer (Ed.), Aplicações Clínicas dos Desenhos Projetivos. São Paulo, SP: Casa do Psicólogo.

Lilienfeld, S. O., Wood, J. M., \& Garb, H. N. (2000). The Scientific Status of Projective Techniques. Psychological Science in the Public Interest, 1(2), 27-66. doi: http://doi.org/10.1111/15291006.002

Meyer, G. J., \& Kurtz, J. E. (2006). Advancing personality assessment terminology: Time to retire "objective" and "projective" as personality test descriptors. Journal of Personality Assessment, 87(3), 223-225. doi: http://doi.org/10.1207/ s15327752jpa8703 01

Meyer, G. J., Viglione, D. J., Mihura, J. L., Erard, R. E., \& Erdberg. (2011). Rorschach Performance Assessment System. Toledo, OH: Rorschach Performance Assessment System.

Noronha, A. P. P., \& Alchieri, J. C. (2004). Knowledge in psychological assessment. Estudos de Psicologia (Campinas), 21(1), 43-52. doi: http:// doi.org/10.1590/S0103-166X2004000100004

Noronha, A. P. P., Beraldo, F. N. de M., \& Oliveira, K. L. de. (2003). Well-know psychological instruments used by psychology students and professional. Psicologia Escolar e Educacional, 7(1), 47-56. doi: http://doi.org/10.1590/S141385572003000100005

Roivainen, E. (2009). A Brief History of the Wartegg Drawing Test. Gestalt Theory, 31(1), 55-71.

Silva, M. C. de V. (1989). TAT: Aplicação e interpretação do Teste de Apercepção Temática. São Paulo, SP: EPU.

Silva, M. C. de V. (2008). O teste de completamento de desenhos de Wartegg (WZT). In A. Elisa \& B. S. G. Werlang (Eds.), Atualizações em méto- 
dos projetivos para avaliação psicológica. São Paulo, SP: Casa do Psicólogo.

Souza, C. V. R. de, Primi, R., \& Miguel, F. K. (2007). Validade do Teste Wartegg: Correlação com 16PF, BPR-5 e desempenho profissional. Avaliação Psicológica, 6(1), 39-49.

Viglione, D. J., Meyer, G. J., Mihura, J. L., Erard, L., \& Erdberg, P. (2012). The Rorschach Performance Assessment System and advances in the Rorschach method. Trabalho apresentado no VI Congresso da Associação Brasileira de Rorschach e Métodos Projetivos. Métodos projetivos e avaliação psicológica: Atualizações, avanços e perspectivas, Brasília, DF.

Villemor-Amaral, A. E. de. (2006). Desafios para a cientificidade das técnicas projetivas. In A. P. P. Noronha, A. A. A. dos Santos, \& F. F. Sisto (Eds.), Facetas do fazer em avaliação psicológica (pp. 163-171). São Paulo, SP: Vetor.

Villemor-Amaral, A. E. de. (2008). A validade teórica em avaliação psicológica. Psicologia: Ciência e Profissão, 28(1), 98-109. doi: http:// doi.org/10.1590/S1414-98932008000100008

Villemor-Amaral, A. E. de. (2009). Métodos Projetos em Avaliações Compulsórias: Indicadores e perfis. In C. S. Hutz (Ed.), Avanços e polêmicas em avaliação psicológica. São Paulo, SP: Casa do Psicólogo.
Villemor-Amaral, A. E. de, \& Pasqualini-Casado, L. (2006). A cientificidade das técnicas projetivas em debate. Psico-USF, 11(2), 185-193. doi: http://doi.org/10.1590/S141382712006000200007

Wartegg, E. (1987). Teste de Wartegg. Diagnóstico de Camadas - WZT. Livros I e II (E. S. von Pflichenfest \& G. M. R. Welter, Trads.). São Paulo, SP: Casa do Psicólogo.

Weiner, I. B. (2000). Princípios da Interpretação do Rorschach (M. C. de V. Moraes, Trad.). São Paulo, SP: Casa do Psicólogo.

Werlang, B. S. G. (2002). TAT, conforme o modelo de Bellak. In J. A. Cunha (Ed.), Psicodiagnóstico-V (pp. 409-415). Porto Alegre, RS: Artmed.

Werlang, B. S. G., \& Cunha, J. A. (1993). Avaliação da personalidade sob enfoque projetivo. In Psicodiagnóstico-R (pp. 123-129). Porto Alegre, $\mathrm{RS}$ : Artes Médicas.
Recebido: 14/03/2017

$1^{a}$ revisão: 09/09/2017 Aceite final: 06/11/2017

(cc)BY (c) O(s) autor(es), 2018. Acesso aberto. Este artigo está distribuído nos termos da Licença Internacional Creative Commons Atribuição 4.0 (http://creativecommons.org/licenses/by/4.0/), que permite o uso, distribuição e reprodução sem restrições em qualquer meio, desde que você dê crédito apropriado ao(s) autor(es) original(ais) e à fonte, fornecer um link para a licença Creative Commons e indicar se as alterações foram feitas. 